Provided for non-commercial research and education use. Not for reproduction, distribution or commercial use.

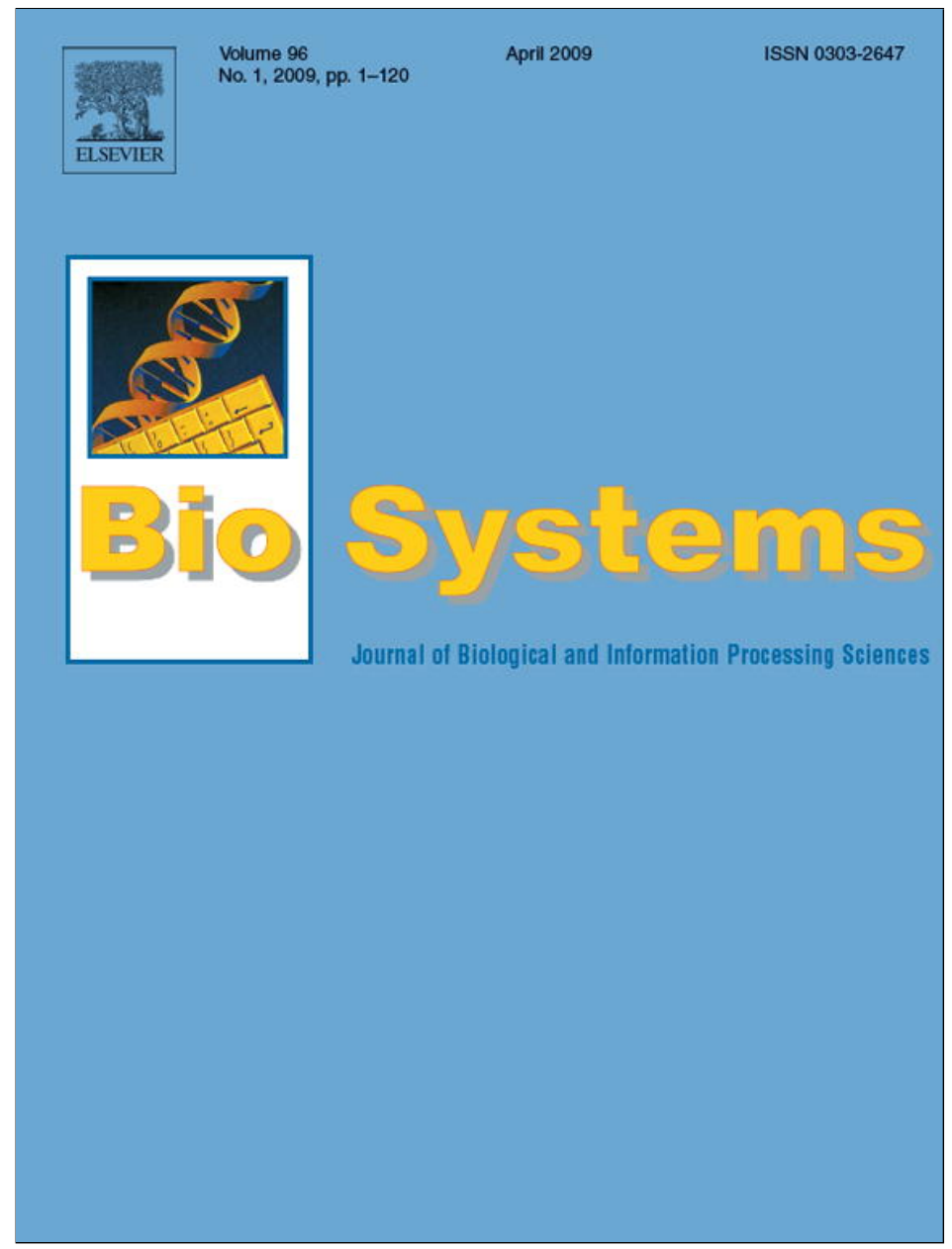

This article appeared in a journal published by Elsevier. The attached copy is furnished to the author for internal non-commercial research and education use, including for instruction at the authors institution and sharing with colleagues.

Other uses, including reproduction and distribution, or selling or licensing copies, or posting to personal, institutional or third party websites are prohibited.

In most cases authors are permitted to post their version of the article (e.g. in Word or Tex form) to their personal website or institutional repository. Authors requiring further information regarding Elsevier's archiving and manuscript policies are encouraged to visit:

http://www.elsevier.com/copyright 


\title{
Evolution of cooperation on dynamical graphs
}

\author{
Ádám Kunn ${ }^{\mathrm{a}, \mathrm{b}, *}$, István Scheuring ${ }^{\mathrm{c}}$ \\ a Department of Plant Taxonomy and Ecology, Institute of Biology, Eötvös University, Pázmány P. sétány 1/C, H-1117 Budapest, Hungary \\ ${ }^{\mathrm{b}}$ Evolution and Ecology Program, International Institute for Applied Systems Analysis, A-2361 Laxenburg, Austria \\ ${ }^{c}$ Department of Plant Taxonomy and Ecology, Research Group of Ecology and Theoretical Biology, Eötvös University and The Hungarian Academy of Sciences, \\ Pázmány P. sétány 1/C, H-1117 Budapest, Hungary
}

\section{A R T I C L E I N F O}

\section{Article history:}

Received 27 August 2008

Received in revised form

19 November 2008

Accepted 20 November 2008

\section{Keywords:}

Game theory

Prisoner's dilemma

Fixation probability

Scale-free network

\begin{abstract}
A B S T R A C T
There are two key characteristic of animal and human societies: (1) degree heterogeneity, meaning that not all individual have the same number of associates; and (2) the interaction topology is not static, i.e. either individuals interact with different set of individuals at different times of their life, or at least they have different associations than their parents. Earlier works have shown that population structure is one of the mechanisms promoting cooperation. However, most studies had assumed that the interaction network can be described by a regular graph (homogeneous degree distribution). Recently there are an increasing number of studies employing degree heterogeneous graphs to model interaction topology. But mostly the interaction topology was assumed to be static. Here we investigate the fixation probability of the cooperator strategy in the prisoner's dilemma, when interaction network is a random regular graph, a random graph or a scale-free graph and the interaction network is allowed to change.

We show that the fixation probability of the cooperator strategy is lower when the interaction topology is described by a dynamical graph compared to a static graph. Even a limited network dynamics significantly decreases the fixation probability of cooperation, an effect that is mitigated stronger by degree heterogeneous networks topology than by a degree homogeneous one. We have also found that from the considered graph topologies the decrease of fixation probabilities due to graph dynamics is the lowest on scale-free graphs.
\end{abstract}

(C) 2008 Elsevier Ireland Ltd. All rights reserved.

\section{Introduction}

While cooperative behaviour is widespread in nature (Dugatkin, 1997), the mechanisms behind its evolution and maintenance are still not fully explored. Population structure has been proposed as one of the mechanism promoting cooperation (Nowak, 2006; Nowak and Sigmund, 2000). Until recently, most studies assumed that the interaction network can be described by a regular graph, which is indeed the case if the players are spatially confined. Ohtsuki et al. (2006) have shown for a number of other interaction topologies that selection favours cooperation (i.e. the fixation probability of a single cooperator is higher than the fixation probability of a neutral mutant) in the prisoner's dilemma game if the benefit $(b)$ of the altruistic act divided by its cost $(c)$ exceeds the average number of neighbours $(k)$, that is, if $b / c>k$. They found this relation to be approximately valid if they use the so called "death-

* Corresponding author at: Department of Plant Taxonomy and Ecology, Institute of Biology, Eötvös University, Pázmány P. sétány 1/C, H-1117 Budapest, Hungary. Tel.: +36 1 3812187; fax: +3613812188

E-mail addresses: kunadam@ludens.elte.hu (Á. Kun), shieazsf@ludens.elte.hu (I. Scheuring). birth" update rule (details see below) in populations of different structure, in which interaction topology is described variously by regular, random regular, random, or scale-free graphs. Recently, Taylor et al. (2007) have proved mathematically that this relation is approximately valid for bi-transitive graphs. Furthermore similar relationship can be derived considering inclusive fitness (Lehman et al., 2007).

Both Ohtsuki et al. (2006) and Taylor and Nowak (2006) have assumed that the graph is static during evolution. This assumption implies that a newborn individual (or accepted strategy-byimitation) in a given position interacts with exactly the same individuals that were connected to every preceding individual at this position. Dispersal from the natal patch (mostly by males) is widespread in nature. Furthermore animal social structure exhibits both rapid changes and long term association (e.g. bottlenose dolphins (Connor et al., 1999); sperm whales (Whitehead, 1995, 1997); long-finned pilot whale (Ottensmeyer and Whitehead, 2003)). Dynamic social connection is characteristic of fission-fusion societies (e.g. chimpanzee (Mitani et al., 2002); spider monkey (Ramos-Fernández, 2005); onager and Grevy's zebra (Sundaresan et al., 2007); African buffalo (Cross et al., 2005); African elephant (Couzin, 1996)). As social networks are not static, the effect of assuming static interaction topology clearly needs attention. Par- 
allely, some recent papers studied the evolution of cooperation on dynamical networks. They either studied the fixation probability of a single cooperator among defectors in the case when graph dynamics is much faster than the dynamics of evolution (Pacheco et al., 2006a), or if the relative speed of graph and evolutionary dynamics were varied systematically they assumed that cooperators and defectors were in the same fraction initially in the population (Pacheco et al., 2006b; Santos et al., 2006a).

Here we investigate how sensitive is the fixation probability of a single cooperator to the network dynamics, if graph dynamics is slow relative to the evolution.

\section{Methods}

In order to allow comparison we considered the same methodology as in Ohtsuk et al. (2006). Namely, a population is considered where the interactions are described by a graph that can vary in time. The population of $N=100^{1}$ individuals consist of defectors and cooperators. An individual derives its payoff, $P$ from interactions with adjacent individuals. A cooperator helps all individuals to whom it is connected thus it pays a cost $(c)$ for each of its neigbour. Neigbours of a cooperator receive the benefit $(b)$. Generally, if a cooperator is connected to $k$ other individuals and of those are cooperators, then its payoff is $b i-c k$. A defector does not provide any help, and therefore has no costs, but it can receive the benefit from neighbouring cooperators. If a defector is connected to $j$ cooperators, then its payoff is $b j$. The fitness of a player is given by $\Phi=1-w+w P$, where $w$ measures the intensity of the selection. Here we assume weak selection where the payoff is small compared to the baseline fitness $(w \ll 1)$. Other possibility is to normalize $P$ by the number of neighbours of the individuals. In this case the beneficial effect of graph degree heterogeneity disappears (Santos and Pacheco, 2005; Szolnoki et al., 2008).

Following Ohtsuki et al. (2006) we employed a "death-birth" updating scheme, where at each update a randomly chosen individual dies; and subsequently the neighbours compete for the empty site in proportion to their fitness. Accordingly, the probability that neighbour $i$ occupies the emptied site is $\Phi_{i} / \sum_{j=1}^{k_{i}} \Phi_{j}$, where the fitness of all neighbours are summed. Alternatively three other update rules can be considered, which is called "birth-death", "imitation" and "pairwise comparison" (for details see Ohtsuki and Nowak, 2006). By using "death-birth", "imitation" or "pairwise comparison" rules evolution of the cooperative strategy is probable if $b / c$ is high enough. However, "birth-death" updating rule has been demonstrated to be detrimental to the evolution of cooperation very close to the ones gained from a well mixed population (see e.g. Ohtsuki and Nowak, 2006, 2008; Ohtsuki et al., 2007).

We have considered three different graph topologies: random regular graphs, random graphs and scale-free graphs. In each case the mean connectivity of the graphs $(k)$ were varied. The graphs were generated as in Ohtsuki et al. (2006), although there are other methods of generating the employed graphs, but we wanted our results to be directly comparable to one of the most eminent prior study in this field. For random regular graphs (RRG), the links between nodes are randomly drawn under the constraint that every node ends up with an equal number of links, $k$. In order to ensure connectedness of the network, every node is first linked to a random node of the already connected ones. Random graphs (RG) are generated in much the same way as RRG, but relaxing the constraint that every node has the same number of links to having $k$ links on average. As for RRG, we first need to make sure that the graph is connected. In a second step two randomly drawn nodes are linked. The second step is repeated until $k N$ links have been distributed. Scale-free networks are generated according to the method of preferential attachment (Albert and Barabási, 2002).

The interaction network was allowed to change. After each update, $\omega$ fraction of the edges belonging to the site being updated was changed. The edge between the focal site and a randomly selected neighbour was exchanged with the edge connecting a randomly chosen site to its neighbour. For example, if $A$ and $B$, and $C$ and $\mathrm{D}$, were originally connected, then after the update, $\mathrm{A}$ is connected to $\mathrm{C}$, and $\mathrm{B}$ is connected to $D$. Because the number of edges belonging to a site never changes, the edge distribution of the graphs remain unchanged (Farkas et al., 2004; Maslov and Sneppen, 2002; Xulvi-Brunet et al., 2003).

In numerical simulations, we then measured the fixation probability of a single cooperator at different levels of graph dynamics $(\omega)$, different average numbers of neighbours $(k)$, and variations in the benefit to cost ratio $(b / c)$. The initial cooperator was placed in a randomly chosen node. For each parameter combination we have constructed 1000 graphs, and on each graph the simulation was repeated 1000 times. The total number of repetitions $(n)$ was thus one million. We computed the numbe of cases $\left(n_{C}\right)$ when the single cooperator spreaded and fixated in the population. Thus we estimated the average fixation probability $p_{C}=n_{C} / n$. Fixation of the cooperative strategy is supported if this probability is higher than the fixation of a neutral mutant, that is if $p_{C}=1 / n$.

\footnotetext{
1 Ohtsuki et al. (2006) have also simulated a population of $N=500$ individuals with similar results as for $N=100$, thus the use of smaller population is justified.
}

\section{Results}

Numerical simulations show that the fixation probability of a cooperator is clearly lower than in the corresponding static graph model, even if the graph dynamics are set to be slow (Fig. 1). The slower the dynamics the bigger the speed of decrease in fixation probability, indicating the sensitivity the results of Ohtsuki et al. (2006) to the assumption of static graphs (Fig. 2). For example, while the $b / c>k$ relation is roughly valid for the static random regular, and random graphs (Fig. 1a and b), it changes approximately to $b / c>(3 / 2) k$, even if only $10 \%$ of the newborn individual's connections change randomly (Fig. 1d and e). Interestingly decreasing of fixation probabilities with increasing graph dynamics is less intense if the interaction structure is described by a scale-free graph comparing to either the random or the random regular graph (Fig. 1c and f). Naturally, it remains still valid that increasing $b / c$ and decreasing $k$ increases the fixation probability of cooperator.

Edge swapping cannot be implemented in regular graphs. Instead, individuals were exchanged to simulate changes in the interaction network (which is not edge swapping per se). Here again, we found that fixation probabilities decreased rapidly with increasing graph dynamics (data not shown). Thus, changes in the interaction network have the same qualitative effect as in the other graphs, even though direct comparison of the regular graph with the others is not possible.

\section{Discussion}

We found that dynamics in the interaction network lowers the fixation probability of the cooperative strategy. It is trivial that in the limit of very fast network dynamic we arrive at the well mixed case, where defection is the only ESS. This limit could have been reached in a qualitatively different way. For example fixation probability drops significantly only at very high values of network dynamic, or it may decrease linearly with increasing network dynamics. These were found to be not the cases, as even a very small amount of dynamic decreased fixation probability significantly. Thus, studying static graphs might lead us to the conclusion that the evolution of cooperation is easy. However, it is not the case in the more realistic setting, where the interaction network is changing, and the linking dynamic is slow and linking is not preferential.

In our investigation the network dynamics was slow and the relinking random. The rate of link change was comparable to the rate of deaths and births of individuals. We employed these slow rates to show that even this causes significant effect. There is evidence that stable association that only change with death and birth do exist in nature (e.g. Karczmarski et al., 2005). This can serve as a base line: there is always some topological change in the interaction network, it cannot be static. Because in most cases generations are overlapping, parents and offspring cannot have exactly the same interaction topology, thus graph dynamic is always present. And even if generations are non-overlapping the environmental fluctuations and stochastic demography will cause change in interaction topology. However, we can ask whether frequency of change in interaction is under evolution? Our results suggest that having zero graph dynamic is evolutionarily more favourable, than having any other amount, assuming that graph topology has not changed and rewiring of graph is random. However, as stated above, this state cannot be realized. Still, the frequency of interaction change is under evolutionarily pressure. However, most of these pressures are not associated with cooperation. For example in fission-fusion societies, interaction network changes, for example, because of temporal change of available food (chimpanzees: Lehmann and Boesch, 2004; Lehmann et al., 2007; African elephants: Wittemeyer et al., 2005; zebras: Rubenstein and Hack, 


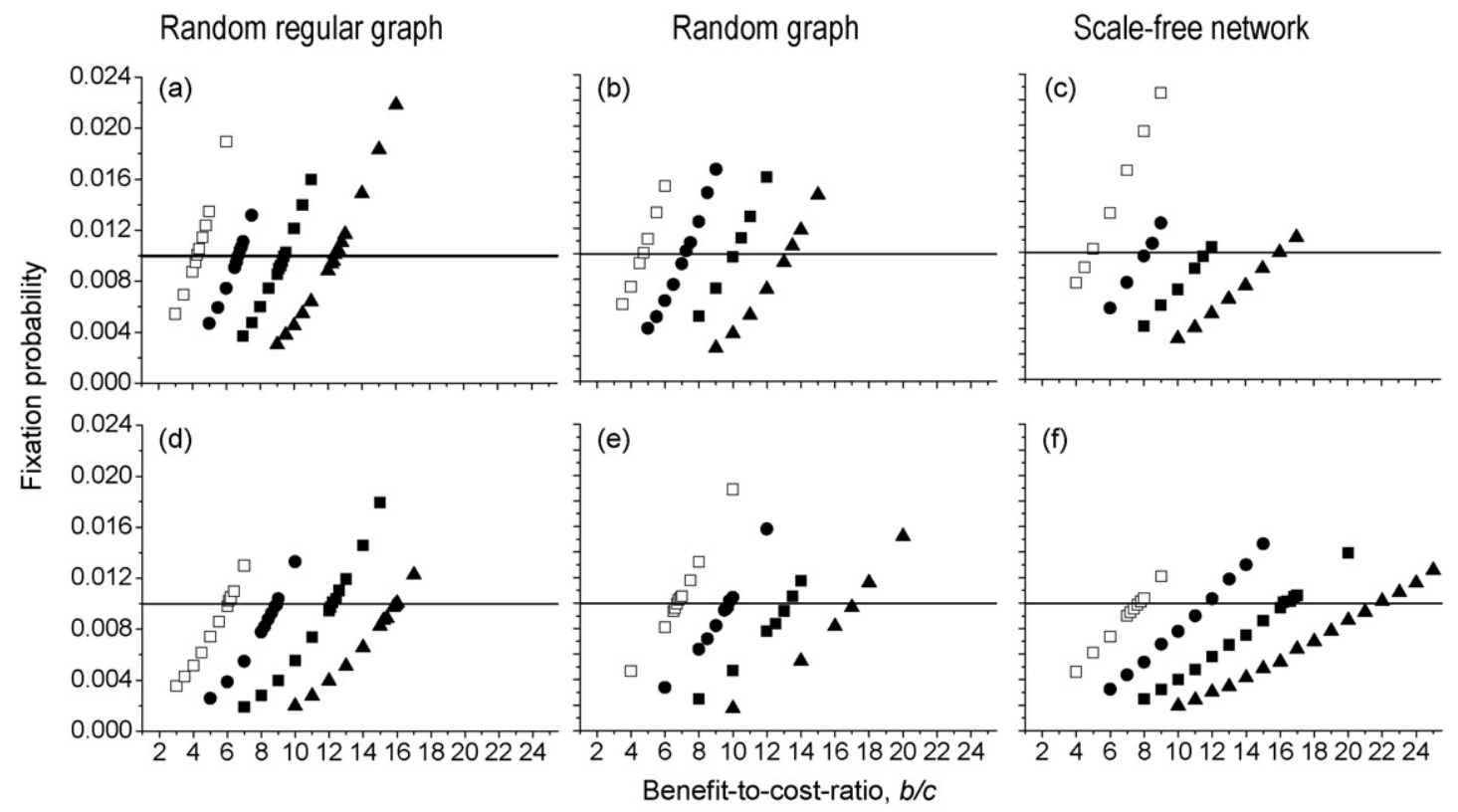

Fig. 1. Fixation probabilities of a single cooperator on static graphs (a-c) and on dynamic graphs (d-f), as a function of the benefit-to-cost ratio. Mean number of neighbours $(k)$ is 4 (open squares), 6 (circles), 8 (filled squares) or 10 (upward triangles). Graph dynamics in (d)-(f) are set to $\omega=0.1$. The strength of selection is $w=0.01$ in all cases.

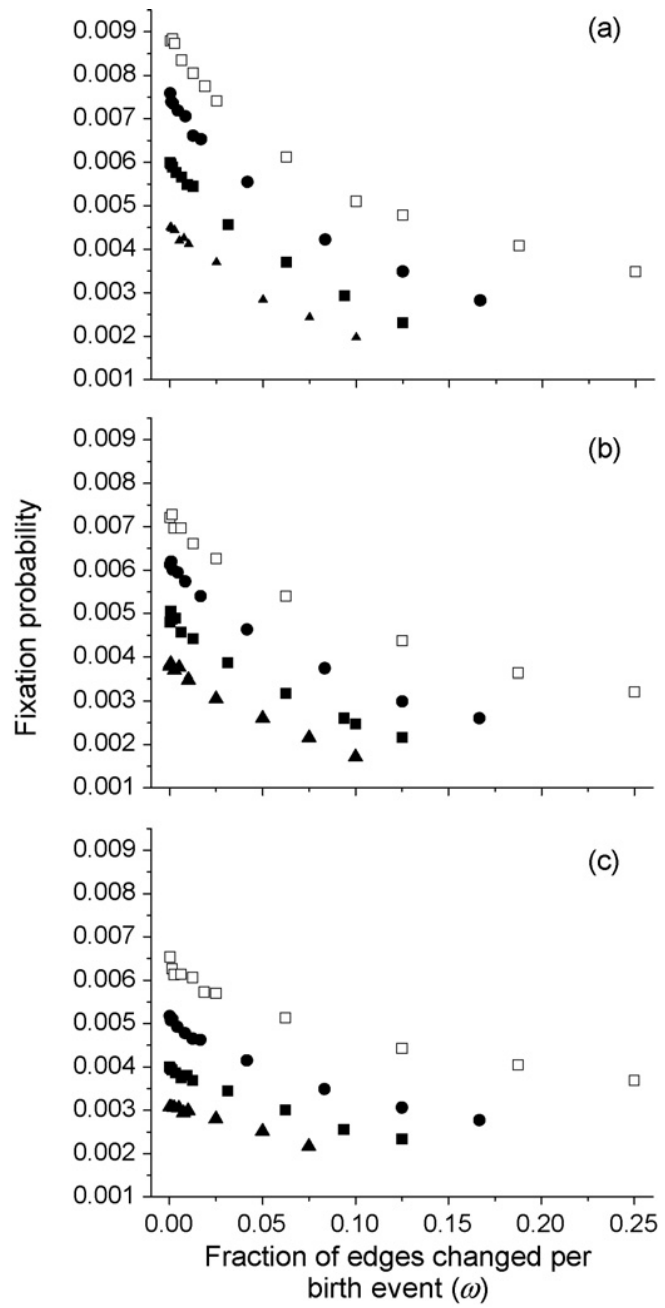

Fig. 2. Fixation probabilities of a single cooperator on dynamic graphs as a function of the intensity of graph dynamics. (a) Random regular graph; (b) random graph; and (c) scale-free graph. Mean number of neighbours $(k)$ is 4 (open squares), 6 (circles), 8 (filled squares) or 10 (upward triangles). The strength of selection is $w=0.01$ and $b / c=k$ in all cases.
2004). Thus there is selection pressure on the behaviour producing fission and the subsequent fusion of the groups.

We employed random relinking in order to focus on the effect of network change. A behaviour which selectively aborts interaction with cheaters and/or search the partnership of cooperators can have positive fitness consequences. Active linking has been shown to benefit cooperation (Pacheco et al., 2006a), but there are two effects in play: that of network change, and that of the preferential choice of individuals to create or to break links with others. Here we have shown that network dynamic alone is harmful to cooperation, and thus we infer that active linking can mitigate its effect (this will be studied in an upcoming paper). The detrimental effect of randomness in partnership was demonstrated by Vukov and Szabó (2004) for a spatial PD with voluntary participation (i.e. there is a third strategy, the loner besides cooperation and defection (Szabó and Hauert, 2002)). In their model a small fraction of the links in the regular network is temporarily replaced by random links. They have found that cooperation decreases with increased randomness in the system, and even a very small amount (around 8\%) of randomization results in loss of the cooperating strategy (Vukov and Szabó, 2004).

We have shown that from the considered graph topologies the decrease of fixation probabilities due to graph dynamics is the lowest on scale-free graphs (Fig. 1). Degree heterogeneity is the highest on the scale-free graph among the employed graphs, and mean fixation probability at a given $b / c$ is the lowest on such graphs (Fig. 1). In accordance with the results of Santos and Pacheco (2005), we have found that fixation probability increased with the degree of the first cooperator node.

Interestingly, a power law distribution was found to describe human cooperation networks (Barabási, 2002; Ebel et al., 2002; Smith, 2002). Lusseau (2003) found scale-free behaviour for large connectivities in a social network of bottlenose dolphins. While population sizes are usually smaller than required for indicating a scale-free degree distribution in social associations, some facts suggest that the structure of animal association network might resemble these graphs. Associations are not random, and certain individuals act as hubs for the society. Older females are identified as hubs in bottlenose dolphins (Lusseau, 2003) and in African elephants (McComb et al., 2001). It seems that certain pigs are more 
"popular" than others (Durell et al., 2004), an observation which was also made for other domestic animals. Spotted hyenas females tend to associate more with higher ranking females (Smith et al., 2007). Degree heterogeneity seems to be common in animal societies; and it promotes cooperation (Santos et al., 2006b). Here we have shown that the scale-free network structure buffers the effect of change in association, which is always present in the network.

\section{Acknowledgements}

We are grateful for the comment of Szabolcs Számadó on earlier draft of the manuscript. Á.K. was a postdoctoral fellow of OTKA (D048406) and currently is a Lisa Meitner Postdoctoral Fellow (M983-N18). I.S. acknowledges support from the Hungarian Research Funds (OTKA T049692, TECT-OTKA NN71700).

\section{References}

Albert, R., Barabási, A.-L., 2002. Statistical mechanics of complex networks. Reviews of Modern Physics 74, 47-97.

Barabási, A.-L., 2002. Linked. Perseus Publishing, Cambridge, MA.

Connor, R.C., Heithaus, M.R., Barre, L.M., 1999. Superalliance of bottlenose dolphins. Nature 397, 571-572.

Couzin, I.D., 1996. Behavioral ecology: social organization in fission-fusion societies. Current Biology 16, R169-R171.

Cross, P.C., Lloyd-Smith, J.O., Getz, W.M., 2005. Disentangling association patterns in fission-fusion societies using African buffalo as an example. Animal Behaviour 69, 499-506.

Dugatkin, L.A., 1997. Cooperation Among Animals: An Evolutionary Perspective. Oxford University Press, New York.

Durell, J.L., Sneddon, I.A., O'Connell, N.E., Whitehead, H., 2004. Do pigs form preferential associations? Applied Animal Behaviour Science 89, 41-52.

Ebel, H., Mielsch, L.-I., Bornholdt, S., 2002. Scale-free topology of e-mail networks Physical Review E 66, 035103(R).

Farkas, I., Derényi, I., Palla, G., Vicsek, T., 2004. Equilibrium statistical mechanics of network structures. Lecture Notes in Physics 650, 163-187.

Karczmarski, L., Würsig, B., Gailey, G., Larson, K.W., Vanderlip, C., 2005. Spinne dolphins in a remote Hawaiian atoll: social grouping and population structure. Behavioral Ecology 16, 675-685.

Lehman, C.L., Keller, L., Sumpter, D.J.T., 2007. The evolution of helping and harming on graphs: the return of the inclusive fitness effect. Journal of Evolutionary Biology 20, 2284-2295.

Lehmann, J., Boesch, C., 2004. To fission or to fusion: effects of community size on wild chimpanzee (Pan troglodytes verus) social organization. Behavioral Ecology and Sociobiology 56, 216.

Lehmann, J., Korstjens, A.H., Dunbar, R.I.M., 2007. Fission-fusion social systems as a strategy for coping with ecological constraints: a primate case. Evolutionary Ecology 21, 613-634.

Lusseau, D., 2003. The emergent properties of a dolphin social network. Proceedings of the Royal Society of London B 270, S186-S188.

Maslov, S., Sneppen, K., 2002. Specificity and stability in topology of protein networks. Science 296, 910-913.

McComb, K., Moss, C., Durant, S.M., Baker, L., Sayialel, S., 2001. Matriarchs as repositories of social knowledge in African elephants. Science 292, 491-494.

Mitani, J.C., Watts, D.P., Muller, M.N., 2002. Recent developments in the study of wild chimpanzee behaviour. Evolutionary Anthropology 11, 9-25.
Nowak, M.A., 2006. Five rules for the evolution of cooperation. Science 314, 1560-1563.

Nowak, M.A., Sigmund, K., 2000. Games on grids. In: Dieckmann, U., et al. (Eds.), The Geometry of Ecological Interactions. Cambridge University Press, Cambridge, UK, pp. 135-150.

Ohtsuki, H., Nowak, M.A., 2006. The replicator equation on graphs. Journal of Theoretical Biology 243, 86-97.

Ohtsuki, H., Nowak, M.A., 2008. Evolutionary stability on graphs. Journal of Theoretical Biology 251, 698-707.

Ohtsuki, H., Pacheco, J.M., Nowak, M.A., 2007. Evolutionary graph theory: breaking the symmetry between interaction and replacement. Journal of Theoretical Biology 246, 681-694.

Ohtsuki, H., Hauert, C., Lieberman, E., Nowak, M.A., 2006. A simple rule for the evolution of cooperation on graphs and social networks. Nature 441,502-505.

Ottensmeyer, C.A., Whitehead, H., 2003. Behavioural evidence for social units in longfinned pilot whales. Canadian Journal of Zoology 81, 1327-1338.

Pacheco, J.M., Traulsen, A., Nowak, M.A., 2006a. Active linking in evolutionary games. Journal of Theoretical Biology.

Pacheco, J.M., Traulsen, A., Nowak, M.A., 2006b. Coevolution of strategy and structure in complex networks with dynamical linking. Physical Review Letters 97, 258103.

Ramos-Fernández, G., 2005. Vocal communication in a fission-fusion society: do spider monkeys stay in touch with close associates? International Journal of Primatology 26, 1077-1092.

Rubenstein, D., Hack, M., 2004. Natural and sexual selection and the evolution of multi-level societies: insight from zebras with comparisons to primates. In: Kappeler, P.M., Van Schaik, C.P. (Eds.), Sexual Selection in Primates: New and Comparative Perspectives. Cambridge University Press, Cambridge.

Santos, F.C., Pacheco, J.M., 2005. Scale-free networks provide a unifying framework for the emergence of cooperation. Physical Review Letters 95, 098104.

Santos, F.C., Pacheco, J.M., Lenaerts, T., 2006a. Cooperation prevails when individuals adjust their social ties. PLoS Computational Biology 2, e140.

Santos, F.C., Pacheco, J.M., Lenaerts, T., 2006b. Evolutionary dynamics of social dilemmas in structured heterogeneous populations. Proceedings of the National Academy of Sciences of the USA 103, 3490-3494.

Smith, J.E., Memenis, S.K., Holekamp, K.E., 2007. Rank-related partner choice in the fission-fusion society of the spotted hyena (Crocuta crocuta). Behavioral Ecology and Sociobiology 61, 753-765.

Smith, R., 2002. Instant messaging as a scale-free network. arXiv:cond-mat 0206378

Sundaresan, S., Fischhoff, I., Dushoff, J., Rubenstein, D., 2007. Network metrics reveal differences in social organization between two fission-fusion species, Grevy's zebra and onager. Oecologia 151, 140-149.

Szabó, G., Hauert, C., 2002. Evolutionary prisoner's dilemma games with voluntary participation. Physical Review E 66, 062903.

Szolnoki, A., Perc, M., Danku, Z., 2008. Towards effective payoffs in the prisoner's dilemma game on scale-free networks. Physica A 387, 2075-2082.

Taylor, C., Nowak, M.A., 2006. Evolutionary game dynamics with non-uniform interaction rates. Theoretical Population Biology 69, 243-252.

Taylor, P.D., Day, T., Wild, G., 2007. Evolution of cooperation in a finite homogeneous graph. Nature $447,469-472$.

Vukov, J., Szabó, G., 2004. Cooperation for volunteering and partially random partnerships. Physical Review E 69, 036107.

Whitehead, H., 1995. Investigating structure and temporal scale in social organisations using identified individuals. Behavioral Ecology 6, 199-208.

Whitehead, H., 1997. Analysing animal social structure. Animal Behaviour 53 1053-1067.

Wittemeyer, G., Douglas-Hamilton, I., Getz, W.M., 2005. The socioecology of elephants: analysis of the processes creating multitiered social structures. Animal Behaviour 69, 1357-1371.

Xulvi-Brunet, R., Pietsch, W., Sokolov, I.M., 2003. Correlations in scale-free networks: tomography and percolation. Physical Review E 68, 036119. 